\title{
An outbreak of Shiga toxin-producing Escherichia coli serogroup 0157 linked to a lamb-feeding event
}

\author{
S. ROWELL ${ }^{1}$, C. KING ${ }^{1}$, C. JENKINS ${ }^{2} *$ T. J. DALLMAN ${ }^{2}$, V. DECRAENE $^{3}$, \\ K. LAMDEN ${ }^{1}$, A. HOWARD ${ }^{4}$, C. A. FEATHERSTONE ${ }^{5}$ AND P. CLEARY ${ }^{3}$ \\ ${ }^{1}$ Cumbria and Lancashire Public Health England Centre, Chorley, Lancashire, UK \\ ${ }^{2}$ Gastrointestinal Bacteria Reference Unit, Public Health England, Colindale, London, UK \\ ${ }^{3}$ Field Epidemiology Service North West, Public Health England, UK \\ ${ }_{5}^{4}$ South Ribble Borough Council, Leyland, Lancashire, UK \\ ${ }^{5}$ Animal and Plant Health Agency, Veterinary Investigation Centre, Thirsk, North Yorkshire, UK
}

Received 6 February 2016; Final revision 29 April 2016; Accepted 25 May 2016; first published online 14 June 2016

\section{SUMMARY}

Fifteen confirmed cases and 15 possible cases of Shiga toxin-producing Escherichia coli (STEC) O157 phage type 21/28 were linked to direct contact with lambs at a 'Lambing Live' event in the North West of England between 29 March and 21 April 2014. Twenty-one (70\%) of the cases were female, $23(77 \%)$ were children aged $<16$ years, of whom $14(46 \%)$ were in the $0-5$ years age group. Five children developed haemolytic uraemic syndrome. Multilocus variable number tandem repeat analysis (MLVA) profiles on 14 human cases were indistinguishable, and 6/10 animal isolates had a MLVA profile identical to the outbreak profile. Whole-genome sequencing analysis revealed that all isolates, both human and animal, fell within a 5-single nucleotide polymorphism cluster indicating the isolates belonged to the same point source. On inspection of the premises, extensive and uncontrolled physical contact between visitors and animals was occuring within the animal pens and during bottle-feeding. Public areas were visibly contaminated with animal faeces. Information to visitors, and the infection control awareness demonstrated by staff, was inadequate. Managing the risk to visitors of STEC O157 infection at animal petting events and open farms requires implementation of stringent control measures by the operator, as outlined in the industry code of practice. Enforcement action is sometimes required to prevent high-risk activities taking place at both permanent and temporary attractions.

Key words: Gastrointestinal infections, public health microbiology, Shiga-like toxin-producing E. coli, veterinary epidemiology and bacteriology.

\section{INTRODUCTION}

Shiga toxin-producing Escherichia coli (STEC) O157 cause gastrointestinal infections associated with a

\footnotetext{
* Author for correspondence: Dr C. Jenkins, Gastrointestinal Bacteria Reference Unit, Public Health England, 61 Colindale Avenue, London NW9 5EQ, UK.

(Email: claire.jenkins@phe.gov.uk)
}

wide spectrum of symptoms ranging from mild, nonbloody diarrhoea, through bloody diarrhoea, to haemolytic uraemic syndrome (HUS). Bloody diarrhoea is seen in $61 \%$ of cases of STEC 0157 in England and HUS develops in $5-15 \%$ of cases, varying by age and sex $[1,2]$. Children aged $<5$ years are the group most at risk of developing STEC-related HUS $[1,2]$. Humans with the infection can excrete STEC O157 for several weeks, with excretion periods 
commonly lasting between 5 days and 1 month [3]. The incubation period (time between having contact with an infectious agent and becoming symptomatic) for diarrhoeal illness caused by infection with STEC O157 is usually $3-4$ days and seldom $<1$ day or $>8$ days, but has been as long as 14 days occasionally $[1,3]$.

Diagnosis is made on the basis of faecal culture. Phage-typing, multilocus variable number tandem repeat analysis (MLVA) and more recently, wholegenome sequencing (WGS), can be undertaken to examine the relationship between isolates and inform outbreak investigations [4-7]. In England, most cases of STEC O157 are recorded as sporadic. The number of cases involved in an outbreak is usually relatively small, with an average of eight cases per outbreak [5].

STEC O157 is found in the gastrointestinal tract of animals and is most frequently detected in cattle, sheep and goats [8-10]. The most common transmission routes of STEC $\mathrm{O} 157$ infection in humans are the consumption of contaminated food (e.g. inadequately cooked meat), faecal-oral spread from humans already infected or contact with animals or their environment. In April 2014 an outbreak of gastrointestinal disease caused by STEC O157 was linked to an animal petting event at a premises located in the North West of England. These premises had no permanent live animal attractions, but ran temporary events on a seasonal basis including, in springtime, an event called 'Lambing Live'. The aim of this report is to describe the outbreak and the public health and microbiological investigations, emphasizing the risks associated with such an event, and to provide examples of how the risks were mitigated.

\section{METHODS}

\section{Epidemiological investigation}

The index case, an 8-year-old child, was reported by the clinical microbiologist at the local hospital to the Health Protection Team (HPT) at Cumbria and Lancashire Public Health England Centre (CLPHEC) on 17 April 2014. The family of the child were interviewed and it was identified that the child had visited the 'Lambing Live' event during their incubation period, where they had direct contact with lambs. On 21 April 2014, CLPHEC were notified of a second case of presumptive STEC O157 in a 2-year-old with HUS. The family confirmed that the child had visited the 'Lambing Live' event on 9
April 2014. Case-finding was initiated by alerting General Practitioners across Lancashire and microbiology departments at local hospitals. Cases, or their family, were interviewed by environmental health staff using the standardized STEC Enhanced Surveillance Questionnaire (https://www.gov.uk/ government/uploads/system/uploads/attachment_data/ file/323423/VTEC_Questionnaire.pdf) to collect demographic and clinical data, and information about potential exposures and sources of infection.

The following case definitions were used:

- A confirmed case visited, or worked at, the premises between 29 March and 21 April 2014 and had STEC O157 isolated from a faecal specimen, or had HUS along with serological evidence of infection with STEC O157.

- A possible case visited, or worked at, the premises between 29 March and 21 April 2014, had gastrointestinal symptoms, but either did not submit a faecal specimen for microbiological testing or their faecal specimen tested negative for STEC O157.

\section{Veterinary and environmental investigation}

From 18 April environmental investigations were carried out by the local authority environmental health team (EHT) and the CLPHEC HPT on a daily basis

The veterinary investigation was undertaken by colleagues from the Animal Health and Veterinary Laboratories Agency (AHVLA) based in Thirsk accompanied by environmental health staff from the EHT on 24 April 2014. Colleagues from AHVLA collected and tested 32 freshly voided floor faecal samples from lambs, and three samples of dried pony faeces using immunomagnetic separation culture methodology [11].

\section{Microbiological investigations}

Serum specimens were sent to Gastrointestinal Bacteria Reference Unit (GBRU) at Public Health England (PHE), Colindale for the detection of antibodies to the lipopolysaccharide of E. coli O157 [12]. Faecal specimens were submitted to the local or regional hospital microbiology laboratories. Isolates of STEC O157 from locally tested specimens were submitted to GBRU for confirmation and typing. At the time of the outbreak, all isolates of STEC O157 submitted to GBRU were typed using MLVA as described previously [5] and WGS, for comparison 
purposes. Isolates with identical MLVA profiles, or with profiles that differed at one locus [single locus variant (SLV)], were considered to be microbiologically linked. Double locus variants (DLVs) were considered to be part of the outbreak only if an epidemiological link existed.

For WGS, DNA was extracted from cultures of STEC O157 for sequencing on the Illumina HiSeq 2500 instrument as described previously [6]. Highquality Illumina reads were mapped to the STEC O157 reference genome Sakai (Genbank accession no. BA000007) using BWA-MEM [13]. Single nucleotide polymorphisms (SNPs) were then identified using GATK2 [14] in unified genotyper mode. Core genome positions that had a high-quality SNP $(>90 \%$ consensus, minimum depth $10 \times$, genotype quality $\geqslant 30$ ) in at least one strain were extracted and RaxML [15] used to derive the maximum-likelihood phylogeny of the isolates.

Genomes were compared to the sequences held in the PHE STEC O157 WGS database. This database comprises genomes from more than 1500 cultures of STEC O157 submitted to GBRU between 1982 and 2015. The majority of isolates were from human cases in England reporting domestically acquired infection, although cases associated with foreign travel and isolates from domestic cattle were also included. Isolates of STEC O157 with $<5$ SNP differences within their core genome are considered closely related and likely to have an epidemiological link [6]. Hierarchical single linkage clustering was performed on the pairwise SNP difference between all isolates at various distance thresholds $(\Delta 250, \Delta 100, \Delta 50, \Delta 25, \Delta 10, \Delta 5, \Delta 0)$. The result of the clustering is a SNP address that can be used to describe the population structure based on clonal groups [6]. Although isolates $>5$ SNPs apart are unlikely to be part of the same temporally linked outbreak, deeper phylogenetic relationships within the 10 or $25 \mathrm{SNP}$ clusters may provide epidemiologically useful information or associations. FASTQ sequences were deposited in the NCBI Short Read Archive under BioProject PRJNA248042.

\section{RESULTS}

\section{Epidemiological investigations}

There were 15 confirmed cases and 15 possible cases of STEC O157 PT21/28 linked to the 'Lambing Live' event, and 18 different households were affected. Of the 30 cases, 29 were visitors to the event and one case worked at the premises and had helped with event. Twenty-one $(70 \%)$ of the cases were female and nine $(30 \%)$ were male. There were seven adults and $23(77 \%)$ children aged $<16$ years of whom 14 $(46 \%)$ were in the $0-5$ years age group. Five children, all aged 2-9 years, developed HUS, of whom three required renal dialysis. There were no fatal cases.

The 'Lambing Live' event took place between 29 March and 21 April 2014. All confirmed cases visited or worked at the site between 8 and 19 April 2014. All the cases became symptomatic between 11 April and 1 May 2014 (Fig. 1) and between 3 and 14 days after visiting or working at the event. As all the cases visited the premises and became unwell during the accepted incubation period for STEC O157 (i.e. 1-14 days), it was not possible to determine which cases were primary (i.e. their infection was acquired at the lambing event) or secondary (i.e. their infection was acquired via person-person contact).

The EHT and the HPT identified several deficiencies in cross-infection control and evidence that the standards of infection control outlined in the industry code of practice [16] had not been adopted, resulting in a failure to adequately control the risk of zoonotic infection to members of the public visiting the premises. Transmission of faecal matter between lambs and visitors was likely to have occurred during the extensive and uncontrolled physical contact between visitors and animals within the animal pens and during bottle-feeding. Public areas, including the handwashing facilities, were visibly contaminated with animal faeces. Information to visitors, staff supervision and the infection control awareness demonstrated by staff was also deemed to be inadequate. Management at the venue were informed of the failures on 18 April 2014 and the proprietor agreed to correct the deficiencies by implementing the recommendations outlined in the industry code of practice [16].

\section{Microbiological investigation of human and animal faecal specimens}

Twenty-three human cases submitted a faecal specimen for microbiological testing, including three cases who also submitted a serum sample. One case submitted a serum sample only. STEC O157 was isolated from 14/23 specimens. One case was faecal culture negative but had HUS and showed serological evidence of recent infection with $E$. coli O157. The remaining eight samples were negative for STEC O157. STEC O157 was isolated from 10/35 animal faecal samples. 


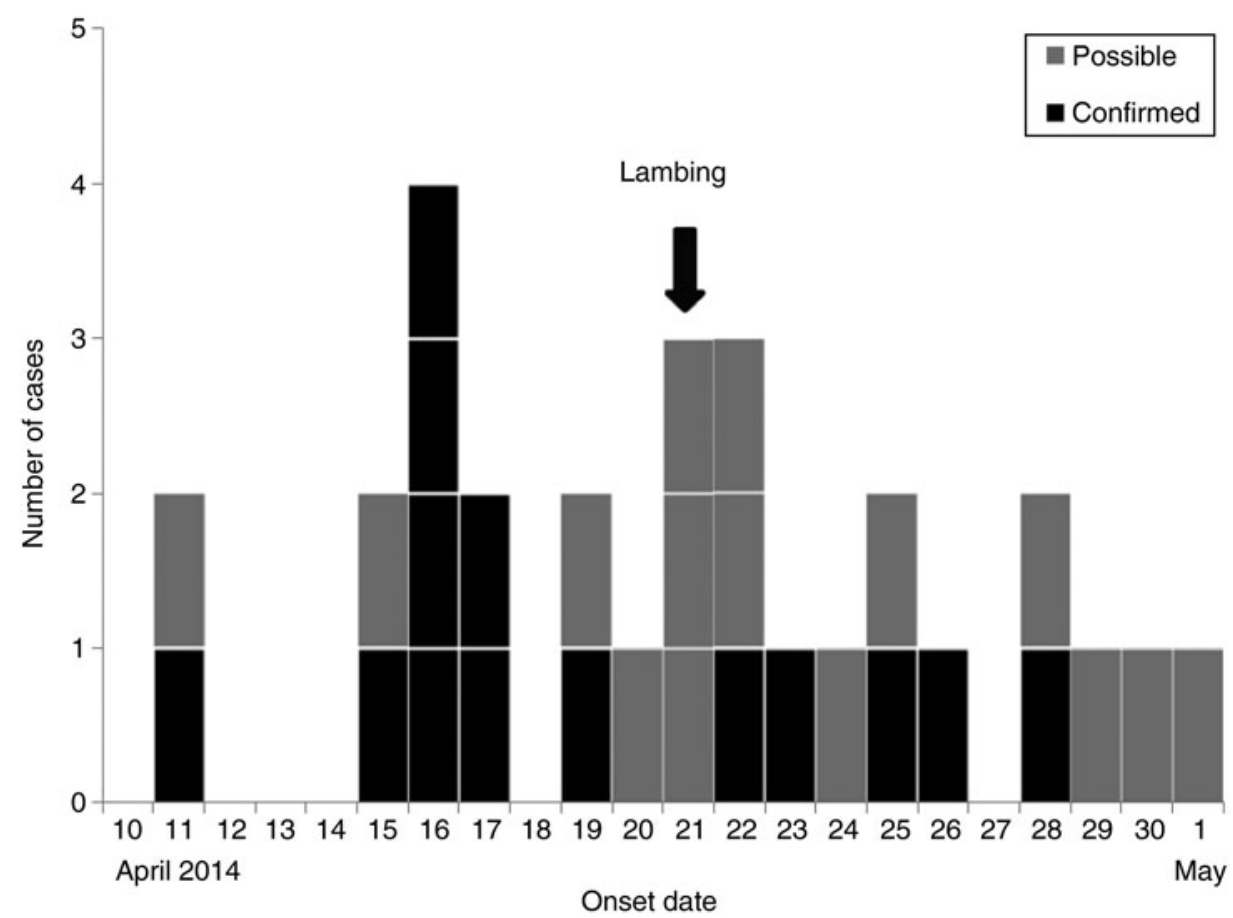

Fig. 1. Distribution of symptomatic STEC O157 cases $(n=29)$ by date of symptom onset during an outbreak associated with a Lambing Live event in Lancashire, April-May 2014.

The 14 isolates from human cases and 10 from the lambs were confirmed as STEC O157 PT21/28 at GBRU. MLVA profiles on the 14 human cases were indistinguishable (identical or one SLV) (Table 1). MLVA was available for 7/10 animal isolates; six had a profile identical to the outbreak profile (8-2-15-5-9-3-7-8) and one had a DLV of the outbreak profile (8-10-14-5-9-3-7-8).

WGS of 13 human and 10 animal isolates showed that they all fell within a 5-SNP cluster (Table 1, Fig. 2). Previous analysis has shown that isolates of STEC O157 that fall within the same 5-SNP cluster are likely to be linked to same point source [6]. The closest isolate in the PHE WGS database was 18 SNPs different, a sporadic case from 2014. Seven of the animal isolates had a SNP not shared with any of the human cases (lambs 3-10), whereas all the SNPs in the remaining two animals (lambs 1 and 2) were also present in all human isolates (Fig. 2). Six children had isolates that had 0 SNP difference from these two animals (cases E, H, I, K, L, M) (Table 1, Fig. 2). Seven human isolates had 1 SNP not shared by any of the animals (cases B, C, D, F, G, J, N), and these isolates are likely to represent unsampled diversity in the lambs handled by these cases at the event (Table 1, Fig. 2).

In addition to the cases described above, one case (case S1 in Table 1) reported in May 2014 had an identical SNP address to the outbreak profile. This case did not visit the 'Lambing Live' event associated with this outbreak but did report handling lambs at a different lambing event (lambing event 2), which was located $\sim 4.5 \mathrm{~km}$ from the premises where the 'Lambing Live' event took place. Investigations revealed that the lambs associated with the 'Lambing Live' event originated from a different, geographically remote farm from the lambs at lambing event 2. Case S1 lived $\sim 5 \mathrm{~km}$ from the farm that supplied the lambs to 'Lambing Live' and although she did not report having any contact with the lambs there, given the proximity of her residence, it is possible that she had contact with their environment. Despite not being able to clearly identify a common source, there are temporal, geographical and exposure similarities and the WGS data indicated a common origin for the isolate from the sporadic isolate and the outbreak isolates was highly likely. The link could be explained by movement of lambs between suppliers prior to both of the lambing events or contact with a contaminated environment.

\section{DISCUSSION}

Open farms and animal contact activities are known to present a risk of STEC O157 infection [11, 17- 
Table 1. Summary of the typing data relating to the human and animal isolates described in this study

\begin{tabular}{|c|c|c|c|c|}
\hline Case identifier & Age, yr & Sex & MLVA & SNP address \\
\hline \multicolumn{5}{|c|}{ Human cases linked to the Lambing Live event } \\
\hline A & 8 & $\mathrm{~F}$ & $8-2-15-5-9-3-7-8$ & Not available \\
\hline $\mathrm{B}$ & 3 & $\mathrm{~F}$ & $8-2-15-5-9-3-7-8$ & $4-4-4-611-879-921-1313$ \\
\hline $\mathrm{C}$ & 21 & $\mathrm{~F}$ & $8-2-15-5-9-3-7-8$ & $4-4-4-611-879-921-2194$ \\
\hline $\mathrm{D}$ & 4 & M & $8-2-15-5-9-3-7-8$ & $4-4-4-611-879-921-1924$ \\
\hline $\mathrm{E}$ & 6 & $\mathrm{~F}$ & $8-1-15-5-9-3-7-8$ & $4-4-4-611-879-921-1111$ \\
\hline $\mathrm{F}$ & 23 & M & $8-2-15-5-9-3-7-8$ & $4-4-4-611-879-921-1978$ \\
\hline G & 2 & M & $8-2-15-5-9-3-7-8$ & $4-4-4-611-879-921-1491$ \\
\hline $\mathrm{H}$ & 3 & $\mathrm{~F}$ & $8-2-15-5-9-3-7-8$ & $4-4-4-611-879-921-1111$ \\
\hline I & 7 & $\mathrm{~F}$ & $8-2-15-5-10-3-7-8$ & $4-4-4-611-879-921-1111$ \\
\hline $\mathrm{J}$ & 13 & $\mathrm{~F}$ & $8-2-15-5-9-3-7-8$ & $4-4-4-611-879-921-1924$ \\
\hline $\mathrm{K}$ & 6 & $\mathrm{~F}$ & $8-2-15-5-9-3-7-8$ & $4-4-4-611-879-921-1111$ \\
\hline $\mathrm{L}$ & 1 & $\mathrm{~F}$ & $8-2-15-5-9-3-7-8$ & $4-4-4-611-879-921-1111$ \\
\hline M & 3 & $\mathrm{~F}$ & $8-2-15-5-9-3-7-8$ & $4-4-4-611-879-921-1111$ \\
\hline $\mathrm{N}$ & 9 & $\mathrm{~F}$ & $8-1-15-5-9-3-7-8$ & $4-4-4-611-879-921-1924$ \\
\hline \multicolumn{5}{|c|}{ Sporadic case (lambing event 2) } \\
\hline $\mathrm{S} 1$ & 6 & $\mathrm{~F}$ & $8-2-15-5-9-3-7-8$ & $4-4-4-611-879-921-1111$ \\
\hline \multicolumn{5}{|c|}{ Animals linked to the Lambing Live event } \\
\hline Lamb 1 & & & $8-2-15-5-9-3-7-8$ & $4-4-4-611-879-921-1111$ \\
\hline Lamb 2 & & & $8-2-15-5-9-3-7-8$ & $4-4-4-611-879-921-1111$ \\
\hline Lamb 3 & & & $8-2-15-5-9-3-7-8$ & $4-4-4-611-879-921-1075$ \\
\hline Lamb 4 & & & $8-2-15-5-9-3-7-8$ & $4-4-4-611-879-921-1315$ \\
\hline Lamb 5 & & & $8-2-15-5-9-3-7-8$ & $4-4-4-611-879-921-1097$ \\
\hline Lamb 6 & & & Not available & $4-4-4-611-879-921-1488$ \\
\hline Lamb 7 & & & $8-2-15-5-9-3-7-8$ & $4-4-4-611-879-921-1075$ \\
\hline Lamb 8 & & & $8-10-14-5-9-3-7-8$ & $4-4-4-611-879-921-1075$ \\
\hline Lamb 9 & & & Not available & $4-4-4-611-879-921-1075$ \\
\hline Lamb 10 & & & Not available & $4-4-4-611-879-921-1315$ \\
\hline
\end{tabular}

MLVA, Multilocus variable number tandem repeat analysis; SNP, single nucleotide polymorphism.

20]. In England, managing this risk is the legal responsibility of the farm or site operator and they should adhere to the guidance laid out in the industry code of practice [16]. This guidance applies to both temporary and permanent attractions and, when correctly applied, will help to mitigate the risks.

Despite the guidance and support available, standards vary between premises. The cause of this outbreak was through contact with lambs, lamb faeces and an environment contaminated with STEC O157 at the lambing event. Outbreaks of STEC O157 specifically linked to direct contact with lambs have been described previously [17, 19]. The outbreak investigation concluded that high-risk activities and deficiencies in infection control at the 'Lambing Live' event had resulted in a high risk of infection to members of the public. This included children having access to pens where the lambs were housed, and the lambs being moved from pens to public areas in order for bottle-feeding to take place. If visitors come into contact with contaminated environments, there is a greater reliance on secondary control measures, such as hand washing. During the 'Lambing Live' event the EHT and the veterinary investigation officer found that equipment such as lamb-feeding bottles were being washed using the hand-washing facilities used by visitors, further increasing the risk of contamination.

Eight of the cases were children that attended nurseries, which are regarded as high-risk settings for secondary cases due to the potential for person-to-person transmission. A strict policy of exclusion and clearance sampling ensured that no secondary cases occurred in these nursery settings or in any other high-risk settings such as food premises.

Both MLVA and WGS analysis provided evidence that the human and animal isolates were genomically linked, although WGS data had a higher level of strain discrimination than MLVA. WGS analysis showed that the human cases were infected with isolates belonging to a subset of the diversity of the isolates detected in the lambs. The meaning of this 


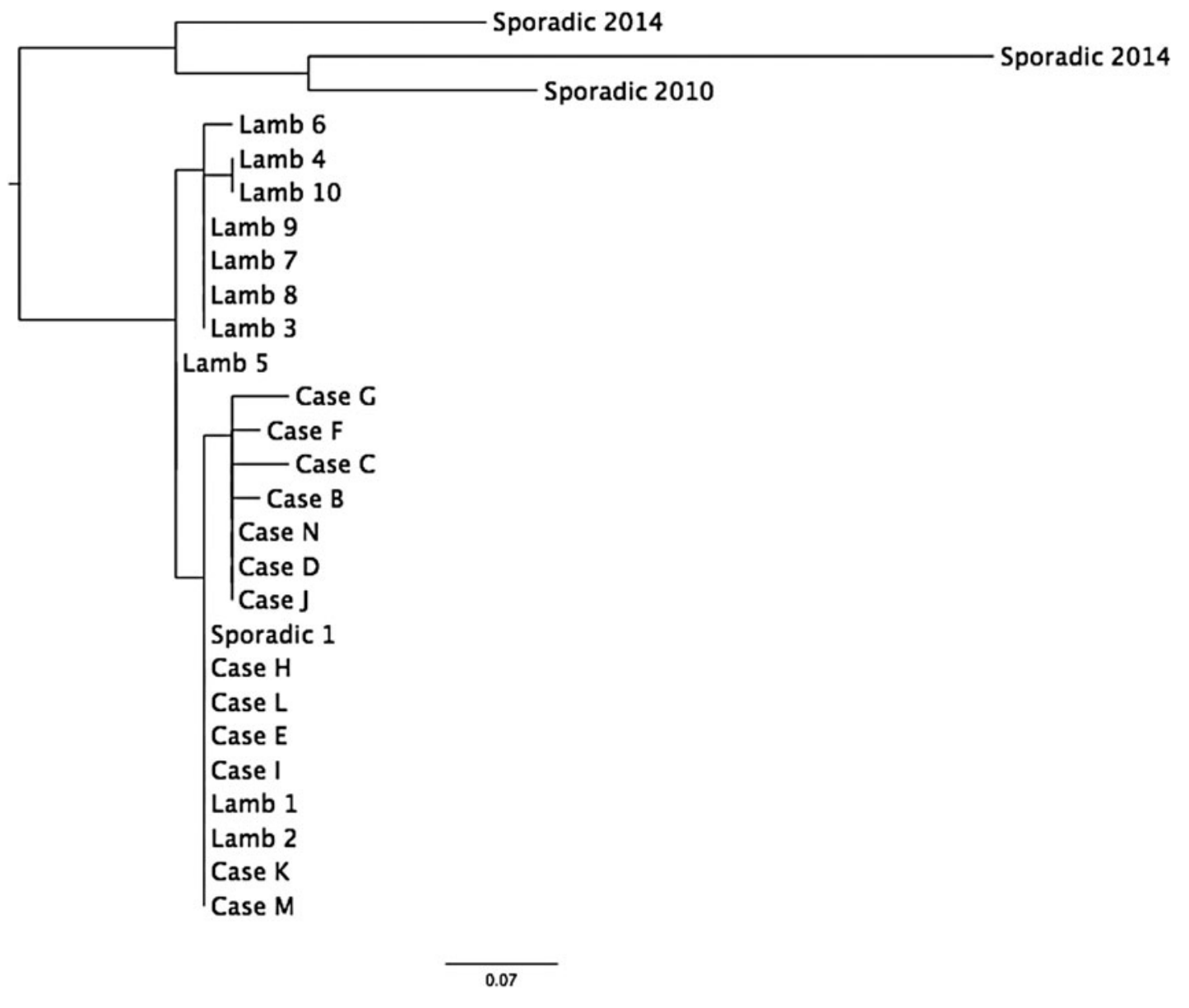

Fig. 2. Maximum-likelihood phylogeny of the isolates from cases and animals (see Table 1 for details).

variation is unclear, as it may result from specific lambs (lambs 1 and 2) being the source of the transmission event or may represent unsampled isolate diversity in either the animals or the human cases. Previous analysis of WGS of STEC O157 from human and animal isolates during a petting farm outbreak in the South East of England in 2009 showed that the isolates differed by 4 SNPs [21]. These authors concluded that this diversity was due to the clonal expansion of a dominant strain via widespread distribution of animal faeces or by multiplication of the outbreak strain in the enviroment prior to the outbreak. A similar level of diversity was observed in both the human and animal isolates sequenced during the outbreak described in the present study.

Despite the higher level of within-outbreak strain diversity exhibited by WGS, variation in the MLVA profiles (SLVs and one DLV) was observed between isolates that had identical SNP addresses (Table 1). This occurs because SNPs (used in WGS analysis) and the polymorphisms of tandemly repeated DNA sequences (used in MLVA) are different biological processes occuring at different rates, possibly under different selection pressures. Previous comparisons of MLVA and SNP analysis during outbreaks have shown that the SNP address is a more robust marker for defining an outbreak than the MLVA profile $[6,7,22]$.

Currently, the cost of WGS one bacterial isolate in-house in the Genome Service \& Development Unit at PHE is $£ 55 \cdot 00$, and this compares favourably to the cost of the battery of phenotypic (biochemistry, serology, phage-typing and antimicrobial resistance typing) and molecular (PCR, pulse-field gel electrophoresis and MLVA) tests required to obtain a similar level of typing data from traditional methods. Since June 2015, all isolates of STEC O157 submitted to GBRU are analysed using WGS for routine public health surveillance and outbreak detection and investigation.

Animal petting events and open farms provide education and enjoyment to large numbers of visitors 
every year. Managing the small risk to visitors of STEC 0157 infection requires implementation of stringent control measures by the farm operator, as outlined in the industry code of practice and consultation with specialist agencies, including local authority environmental health teams. The risk of cross-infection to members of the public cannot be entirely eliminated and in instances of non-compliance enforcement action may be required to prevent higrisk activities taking place at both permanent and temporary attractions.

\section{ACKNOWLEDGEMENTS}

We thank Marie Anne Chattaway, Neil Perry, Vivienne do Nascimento and Yoshini Taylor at GBRU and Lisa Byrne, Naomi Launders and Kirsten Glen in the Gastrointestinal Infections Department. We also acknowledge everyone who was part of the Outbreak Control Team including the Animal and Plant Health Agency. This work was supported by the National Institute for Health Research Health Protection Research Unit in Gastrointestinal Infections. The views expressed are those of the author(s) and not necessarily those of the NHS, the NIHR, the Department of Health or Public Health England.

\section{DECLARATION OF INTEREST}

None.

\section{REFERENCES}

1. Byrne L, et al. The epidemiology, microbiology and clinical impact of Shiga toxin-producing Escherichia coli in England, 2009-2012. Epidemiology and Infection 2015; 143: 3475-3487.

2. Launders $\mathbf{N}$, et al. Disease severity of Shiga toxinproducing $E$. coli $\mathrm{O} 157$ and factors influencing the development of typical haemolytic uraemic syndrome: a retrospective cohort study, 2009-2012 BMJ Open 2016; 6: e009933.

3. Hawker JE. Communicable Disease Control and Health Protection Handbook. Chichester: John Wiley \& Sons, 2012.

4. Khakhria R, Duck D, Lior H. Extended phage-typing scheme for Escherichia coli O157:H7. Epidemiology and Infection 1990; 105: 511-520.

5. Byrne $\mathbf{L}$, et al. Evaluating the use of multilocus variable number tandem repeat analysis of Shiga toxinproducing Escherichia coli $\mathrm{O} 157$ as a routine public health tool in England. PLoS ONE 2014; 9: e85901.

6. Dallman TJ, et al. Whole-genome sequencing for national surveillance of Shiga toxin-producing Escherichia coli O157. Clinical Infectious Disease 2015; 61: 305-312.
7. Jenkins $\mathbf{C}$, et al. public health investigation of two outbreaks of shiga toxin-producing Escherichia coli $\mathrm{O} 157$ associated with consumption of watercress. Applied and Environmental Microbiology 2015; 81: 3946-3952.

8. Ogden ID, MacRae M, Strachan NJ. Concentration and prevalence of Escherichia coli $\mathrm{O} 157$ in sheep faeces at pasture in Scotland. Journal Applied Microbiology 2005; 98: 646-651.

9. Chase-Topping M, et al. Super-shedding and the link between human infection and livestock carriage of Escherichia coli O157. Nature Reviews Microbiology 2008; 6: 904-912.

10. La Ragione RM, et al. Escherichia coli $\mathrm{O} 157: \mathrm{H} 7$ colonization in small domestic ruminants. FEMS Microbiology Reviews 2009; 33: 394-410.

11. Pritchard GC, et al. Verocytotoxigenic Escherichia coli O157 in animals on public amenity premises in England and Wales, 1997 to 2007. Veterinary Record 2009; 164: 545-549.

12. Chart H, Cheasty T. Human infections with verocytotoxin-producing Escherichia coli O157-10 years of E. coli $\mathrm{O} 157$ serodiagnosis. Journal of Medical Microbiology 2008; 57: 1389-1393.

13. Li H, Durbin R. Fast and accurate long-read alignment with Burrows-Wheeler transform. Bioinformatics 2010; 26: 589-595.

14. McKenna A, et al. The Genome Analysis Toolkit: a MapReduce framework for analyzing next-generation DNA sequencing data. Genome Research 2010; 20: 1297-1303.

15. Stamatakis A, RAxML version 8: a tool for phylogenetic analysis and post-analysis of large phylogenies. Bioinformatics 2014; 30: 1312-1313.

16. Farming and Countryside Education. Preventing or controlling ill health from animal contact at visitor attractions, 2012 (http://www.faceonline.org.uk/resources/ preventing-or-controlling-ill-health-from-animal-contactatvisitor-attractions-industry-code-of-practice).

17. Milne LM, et al. Escherichia coli O157 incident associated with a farm open to members of the public. Communicable Disease and Public Health 1999; 2: 22-26.

18. Crump JA, et al. An outbreak of Escherichia coli O157: H7 infections among visitors to a dairy farm. New England Journal Medicine 2002; 347: 555-560.

19. Ihekweazu C, et al. Large outbreak of verocytotoxinproducing Escherichia coli $\mathrm{O} 157$ infection in visitors to a petting farm in South East England, 2009. Epidemiology and Infection 2012; 140: 1400-1413.

20. Rechel B, et al. Investigation of a spatiotemporal cluster of verotoxin-producing Escherichia coli O157 infections in eastern England in 2007. Eurosurveillance 2011; 16(28).

21. Underwood AP, et al. Public health value of nextgeneration DNA sequencing of enterohemorrhagic Escherichia coli isolates from an outbreak. Journal Clinical Microbiology 2013; 51: 232-237.

22. Butcher $\mathbf{H}$, et al. Whole genome sequencing improved case ascertainment in an outbreak of Shiga toxinproducing Escherichia coli $\mathrm{O} 157$ associated with raw drinking milk. Epidemiology \& Infection (in press). 